\title{
Simulating the Shastry-Sutherland Ising Model Using Quantum Annealing
}

\author{
Paul Kairys $\odot,{ }^{1,2}$ Andrew D. King $\odot,{ }^{3}$ Isil Ozfidan, ${ }^{3}$ Kelly Boothby $\odot,{ }^{3}$ Jack Raymond, ${ }^{3}$ \\ Arnab Banerjee, ${ }^{4,5,{ }^{*}}$ and Travis S. Humble $\odot^{1,2, \dagger}$ \\ ${ }^{1}$ Bredesen Center for Interdisciplinary Research and Graduate Education, University of Tennessee, Knoxville, \\ Tennessee, USA \\ ${ }^{2}$ Quantum Computing Institute, Oak Ridge National Laboratory, Oak Ridge, Tennessee, USA \\ ${ }^{3}$ D-Wave Systems, Inc., Burnaby, British Columbia, Canada \\ ${ }^{4}$ Neutron Sciences Division, Oak Ridge National Laboratory, Oak Ridge, Tennessee, USA \\ ${ }^{5}$ Department of Physics and Astronomy, Purdue University, West Lafayette, Indiana, USA
}

(Received 21 August 2020; accepted 23 November 2020; published 14 December 2020)

\begin{abstract}
A core concept in condensed matter physics is geometric frustration that leads to emergent spin phases in magnetic materials. These distinct phases, which depart from the conventional ferromagnet or the antiferromagnet, require unique computational techniques to decipher. In this study, we use the canonical Ising Shastry-Sutherland lattice to demonstrate new techniques for solving frustrated Hamiltonians using a quantum annealer of programmable superconducting qubits. This Hamiltonian can be tuned to produce a variety of intriguing ground states ranging from short- and long-range orders and fractional order parameters. We show that a large-scale finite-field quantum annealing experiment is possible on 468 logical spins of this model embedded into the quantum hardware. We determine microscopic spin configurations using an iterative quantum annealing protocol and develop mean-field boundary conditions to attenuate finitesize effects and defects. We not only recover all phases of the Shastry-Sutherland Ising model —including the well-known fractional magnetization plateau in a longitudinal field - but also predict the spin behavior at the critical points with significant ground-state degeneracy and in the presence of defects. The results lead us to establish the connection to the diffuse neutron scattering experiments by calculation of the static structure factors.
\end{abstract}

DOI: 10.1103/PRXQuantum.1.020320

\section{INTRODUCTION}

Frustration is an essential feature for the exotic behavior of several magnetic materials and occurs when the geometry of the system prohibits all local constraints in the microscopic Hamiltonian being satisfied simultaneously. This frustration leads to ground-state degeneracy and even the emergence of novel phases of matter. Antiferromagnetic spins on a triangular lattice form the simplest frustrated geometry in two dimensions and extrapolate to a large collection of geometrically frustrated lattices - including the Kagome and triangular lattices - promising a variety of emergent, unconventional phases of matter such as spin liquids [1], spin ices [2,3], and stripe phases [4]. Among

\footnotetext{
*arnabb@purdue.edu
}

†humblets@ornl.gov

Published by the American Physical Society under the terms of the Creative Commons Attribution 4.0 International license. Further distribution of this work must maintain attribution to the author(s) and the published article's title, journal citation, and DOI. these, the geometrically frustrated Shastry-Sutherland [5] model, with Hamiltonian

$$
H=J_{1} \sum_{\langle i, j\rangle} \sigma_{(i)}^{z} \sigma_{(j)}^{z}+J_{2} \sum_{\langle i, j\rangle} \sigma_{(i)}^{z} \sigma_{(j)}^{z}+h_{z} \sum_{i} \sigma_{(i)}^{z}
$$

defined over the lattice shown in Fig. 1(a), is one in a rare class of exactly soluble geometrically frustrated Hamiltonians. The frustration originates from incompatibility between the nearest-neighbor $J_{1}$ interactions on a square sublattice and the next-nearest-neighbor $J_{2}$ interactions, which form triangular motifs on the square grid. The Ising Shastry-Sutherland model exhibits multiple phases, including a trivial ferromagnetic (FM) phase when $h_{z} \gg$ $J_{1}, J_{2}$, a Néel antiferromagnetic (AFM) phase when $J_{1} \gg$ $h_{z}, J_{2}$, and a more interesting AFM spin-dimer phase when $J_{2} \gg h_{z}, J_{1}$ [6]. Intriguingly, for $J_{1} \approx J_{2}>0$, a nontrivial phase arises in which the magnetic susceptibility shows a $\frac{1}{3}$ magnetization plateau. This plateau arises from a sixfold spin degenerate solution when above a critical longitudinal field strength [6-8]. 
(a)

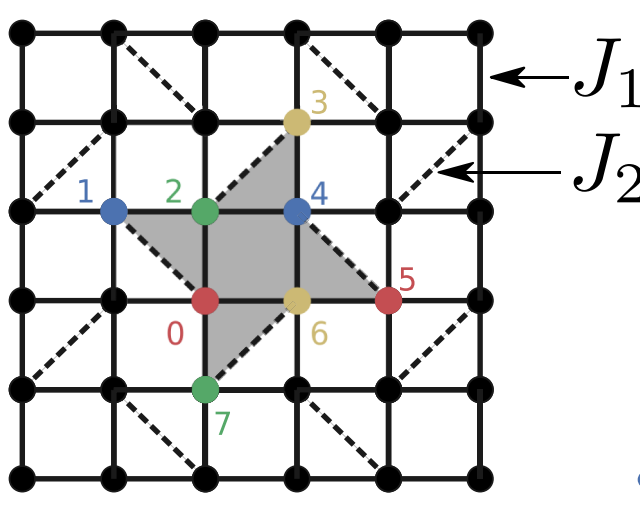

(c)

(b)
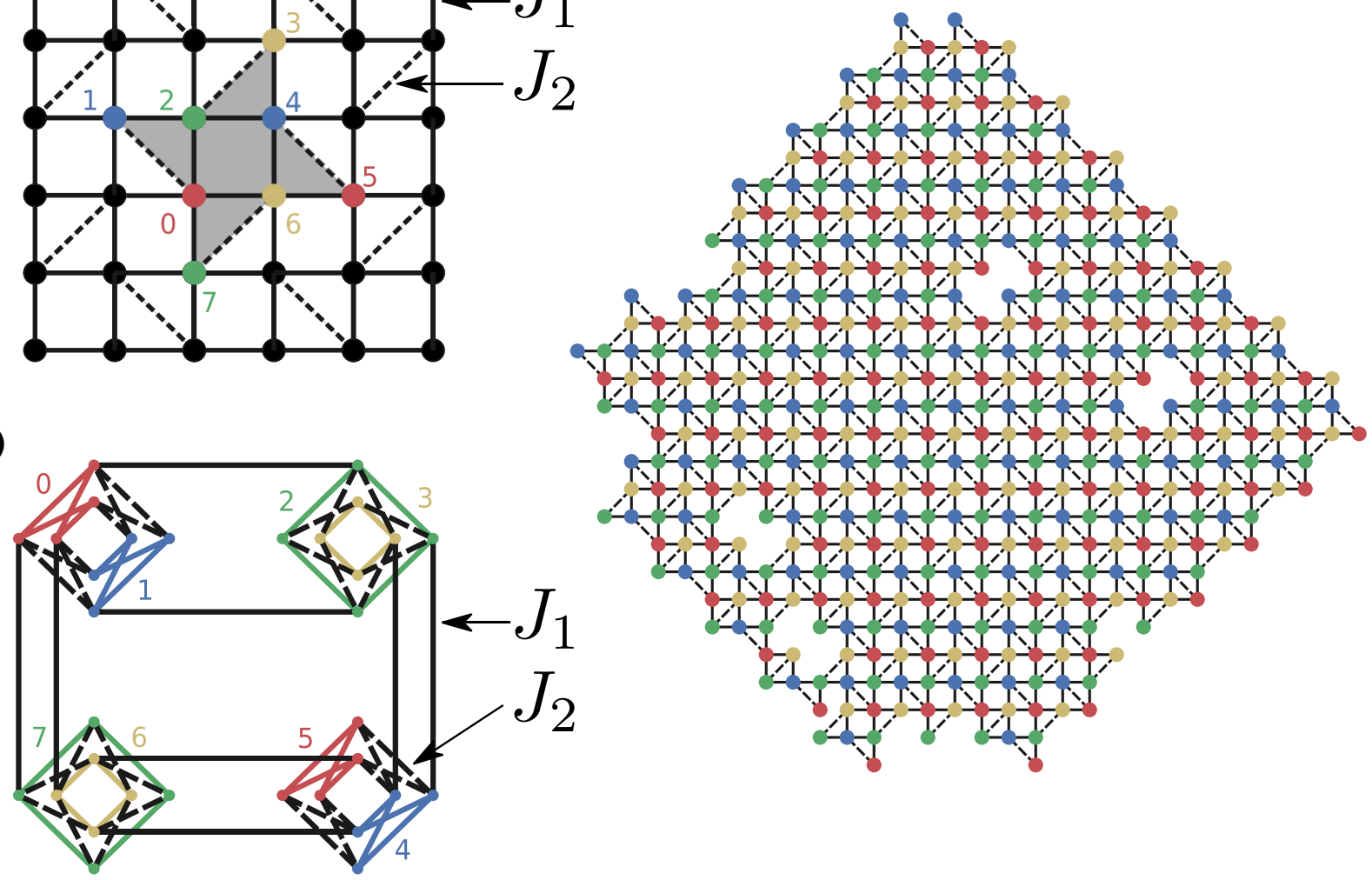

FIG. 1. Embedding the Shastry-Sutherland lattice in the D-Wave quantum annealer. (a) The Shastry-Sutherland lattice showing the nearest-neighbor square (solid black lines) and the next-nearest-neighbor dimer (dashed black lines) interactions. Highlighted in gray is the logical unit cell with eight spins labeled. (b) The embedding of the logical unit cell from (a) into a Chimera graph (full embedded graph shown in Fig. 5). This "half-cell" embedding maps each logical spin to a chain of four ferromagnetically connected qubits (solid color lines) and each dimer pair to a single Chimera unit cell. Each logical square bond is mapped to one external coupler (solid black lines) between Chimera unit cells and each dimer bond is mapped to eight internal couplers (dashed black lines) within a Chimera unit cell. This embedding preserves the symmetry of the logical problem and ensures that every physical qubit experiences similar local environments, which prevents chain breaking. (c) The embedding of a 468-spin lattice in the quantum annealer. Missing lattice sites in the underlying Chimera graph reveal defects that originate from defective qubits or bonds in the annealer. Effects from these defects, as well as from finite boundaries, are mitigated by applying self-consistent mean-field boundary conditions (see the text). These boundary conditions are applied on both the external boundaries and the internal boundaries (qubits surrounding defects) simultaneously.

The exact solubility of the Shastry-Sutherland models and unique magnetization plateaus have generated significant interest in the physics community, stoked by the fact that the Ising Shastry-Sutherland model and its variations may explain the magnetism in real materials, such as the rare-earth tetraborides $\left(R \mathrm{~B}_{4}\right)$, which exhibit several magnetic plateaus with fractional magnetization [6,8-10]. Existing literature suggests that sampling ground states of this model is not computationally hard in the noncritical phases, but there has not been a detailed study on the ground-state magnetic ordering near the critical points and the computational complexity of sampling ground states near critical points [7]. The square topology, unique magnetic physics, and potential material realizations of this model make a compelling testbed to evaluate the efficacy of quantum annealing (QA) to address fundamental material physics and present new paths for the crossexamination of simulation and experimental data.

Determining low-energy microscopic spin ensembles using QA relies on fundamentally different principles as compared to conventional classical computing methods. Whereas traditional simulated annealing uses thermal excitations and the ergodic principle to find the energetic ground state [11], quantum annealing uses a quantum tuning parameter such as a transverse magnetic field to drive transitions between quantum states $[12,13]$. The dynamics induced by the transverse field allows QA to explore the energy landscape, potentially faster than classical approaches [14].

We use a programmable quantum annealer realized by a two-dimensional lattice of superconducting qubits. This device implements a transverse-field Ising model (TFIM) 
defined by [13]

$$
\begin{aligned}
H(s)= & A(s) \sum_{i} \sigma_{(i)}^{x} \\
& +B(s)\left[\sum_{i} h_{i} \sigma_{(i)}^{z}+\sum_{\langle i, j\rangle} J_{i j} \sigma_{(i)}^{z} \sigma_{(j)}^{z}\right],
\end{aligned}
$$

where amplitudes $A(s)$ and $B(s)$ determine the relative strength of the transverse field and Ising terms and are controlled through the dimensionless annealing parameter $s \in[0,1]$ representing the depth of the anneal. These amplitudes are smooth functions of $s$ with $A(0) \gg B(0)$ and $A(1) \ll B(1)$. The parameters $J_{i j}$ and $h_{i}$ define tunable Ising interactions and a per-qubit tunable longitudinal magnetic field, respectively, which enables the realization of various magnetic systems and lattice geometries within the processor. The available couplers are arranged in a "Chimera" graph [15], used in several recent examples to validate material simulations using QA. This includes the simulation of the three-dimensional (3D) spin-glass transition [16] and the demonstration of the BerezinskiiKosterlitz-Thouless transition in a two-dimensional TFIM [17].

We examine the behavior of the Ising ShastrySutherland model across all its phases by sampling the low-energy microscopic states using QA. Our approach goes beyond conventional QA approaches by using an iterative simulation protocol known as quantum evolution Monte Carlo (QEMC) chaining [14,17] to sample the ground-state manifold of the frustrated Ising Hamiltonian. In order to accurately simulate the bulk behavior, we also apply a technique for mean-field boundary conditions $[18,19]$ to mitigate finite-size effects as well as the presence of defects in the quantum annealer. The microscopic ensemble of states obtained from QA allows us to compute the phase diagram for the frustrated Hamiltonian as well as its static structure factor - observables directly accessed in material experiments [9]. These results establish the use of programmable quantum annealing as an avenue to understanding frustration in a magnetic Hamiltonian with the potential to simulate the behaviors of two-dimensional spintronic materials for future experimental comparison.

\section{ANNEALING METHODS}

We start by mapping the Shastry-Sutherland Hamiltonian, defined in Eq. (1) and shown in Fig. 1(a), into the native architecture on a D-Wave $2000 \mathrm{Q}^{\mathrm{TM}}$ quantum annealer [20], known as a Chimera graph. The embedding of the Hamiltonian into the processor uses strongly coupled ferromagnetic chains to encode each logical spin site [21]. Shown in Fig. 1(b), the embedding encodes a single logical spin to a cyclic chain of four physical qubits, each logical dimer bond to eight physical internal couplers, and each logical square bond to one physical external coupler. This so-called "half-cell" embedding exploits the symmetries of the Shastry-Sutherland lattice and the Chimera graph to reinforce spatial uniformity, i.e., dimer bond maps to internal couplers and square bonds map to external couplers (see Appendix A). This scheme results in a large lattice of 468 logical spins from the 1872 functioning qubits, as shown in Fig. 1(c).

The limited sizes of near-term quantum devices, and the presence of defects pose a challenge to calculating results for materials in the thermodynamic limit. Here we apply self-consistent mean-field boundary conditions [19] in the embedded Hamiltonian by leveraging the independent control over the longitudinal magnetic fields on each qubit. Specifically, we iteratively determine boundary magnetic fields such that the magnetization is uniform and translationally invariant (see Appendices B-D for all calibration methods used). This is an effective technique to screen away the defects and boundaries and preserve the integrity of the Hamiltonian, allowing us to observe microscopic structures found in the thermodynamic limit, which we present in subsequent sections.

The third major challenge in applying QA technologies is limited control over device dynamics. Conventional QA of Eq. (2) relies on controlling the amplitudes $A(s)$ and $B(s)$ through an adiabatic evolution from $s=0$ to $s=1$ to prepare ground states. However, the adiabatic condition is difficult to satisfy due to limits on device control, qubit coherence, and other factors [22]. To overcome this, we use the technique of QEMC chaining $[14,17]$ that relaxes the system through an iterative sequence of equilibrations at a fixed transverse-field strength. This method has two parameters, $s_{p}$ and $t_{p}$, which control the magnitude of the transverse field (i.e., the quantum tunneling energy) in Eq. (2) and the equilibration time, respectively (see Appendix E). This unconventional QA protocol allows us to mitigate the limitations of adiabatic QA by once again taking advantage of the programmable control features of annealing devices. In the next section we lay out a protocol to determine optimal QEMC parameters for an embedded spin system.

An essential step towards the optimal performance of the QEMC protocol is determining the values of $s_{p}$ and $t_{p}$ (pause and equilibration times) that produce the lowestenergy classical ground states in the fewest iterations. In Fig. 2(a) we show that by probing different $s_{p}$ values we can determine that $s_{p}=0.4$ produces the lowest-energy classical ground states upon equilibration. Empirically, this value signifies the right balance between tunneling dynamics and classical energy (see Appendix E). Under these conditions, the system is able to converge from an initial state far from the solution to the optimal magnetization in a few iterations. An example is demonstrated in Fig. 2(b) where the system anneals from an initial FM configuration to the optimal magnetization of $\frac{1}{3}$ in a single iteration; 
(a)

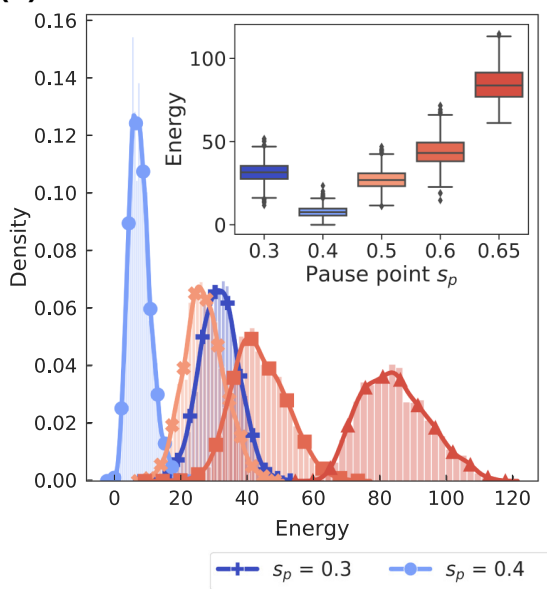

(b)

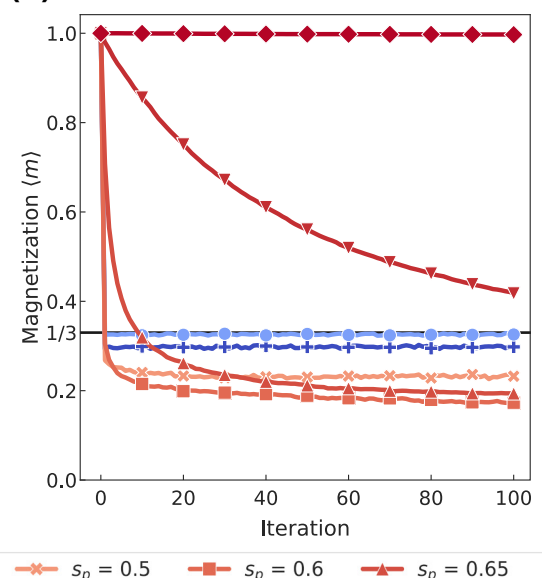

(c)

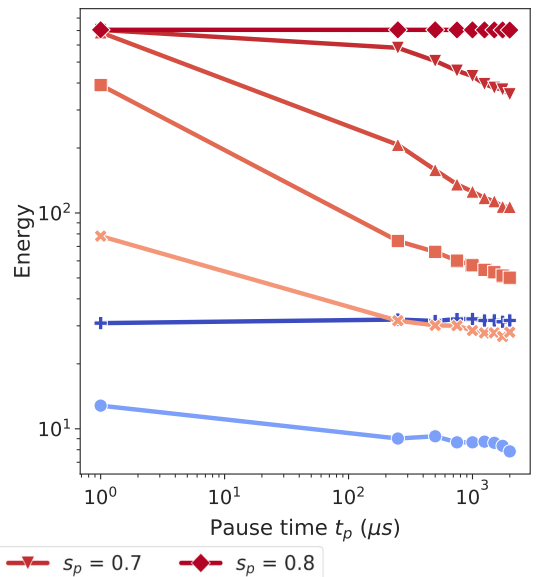

FIG. 2. Optimal QEMC annealing parameters. (a) Simulated energy distributions using QEMC chains at $\left(J_{1}=1, J_{2}=1, h_{z}=2.1\right)$ with $t_{r}=1 \mu \mathrm{s}$ ramps and $t_{p}=1998 \mu \mathrm{s}$. The energies displayed are rescaled according to the lowest-energy configuration observed in experiment. Two values, $s_{p}=0.7, s_{p}=0.8$, are omitted from (a) for clarity because $\left\langle H\left(s_{p}=0.7\right)\right\rangle \geq 600$ and $\left\langle H\left(s_{p}=0.8\right)\right\rangle \geq 700$ lie far outside of the domain shown in (a). The inset is an alternative view of (a) that clearly shows the parabolic energy curve as a function of $s_{p}$ with a minimum at $s_{p}=0.4$. (b) The convergence of the magnetization from a ferromagnetic state at $\left(J_{1}=1, J_{2}=1, h_{z}=2.1\right)$ with $t_{r}=1 \mu \mathrm{s}$ ramps and $t_{p}=1998 \mu \mathrm{s}$ to the $\frac{1}{3}$ magnetization plateau (shown in black). For $s_{p}<0.5$, convergence to the bulk magnetization occurs in less than 5 iterations; however, these states are not energetically optimal and convergence to the correct microscopic order takes many tens of iterations. (c) The average returned energy as a function of $s_{p}$ and $t_{p}$ with $t_{r}=1 \mu$ s. Unless $s_{p}$ is sufficiently strong such that the timescale of tunneling dynamics is much shorter than the anneal time, increasing the pause time has a dramatic effect in finding low-energy solutions.

however, it takes 40-50 QEMC iterations to converge to the appropriate microscopic order. In Fig. 2(c) we show that increasing the value of $t_{p}$ leads to significantly lower energy and therefore we choose the maximum equilibration time of $t_{p}=1998 \mu \mathrm{s}$ for our experiments. These optimal annealing parameters allow us to rapidly arrive at accurate low-energy states without having to satisfy the adiabatic condition - a key impediment in traditional QA.

\section{SIMULATION RESULTS}

Determining the optimal annealing parameters permit us to confidently explore the low-energy solutions for a large parameter space of the microscopic Hamiltonian, Eq. (1). We simulated an ensemble of low-energy states for the Shastry-Sutherland Ising model over the range of parameters $h_{z} \in[-8,8]$ and $J_{2} / J_{1} \in[0,3]$, from which we computed the phase diagram shown in Fig. 3(a). The results not only identified all four long-range ordered phases - including the $\frac{1}{3}$-magnetization plateau, for which a cross-sectional plot is shown in Fig. 3(b) - but we also obtained excellent agreement with the expected location of the phases and phase transitions across the entire parameter range, indicated by the overlapping theoretical predictions in Fig. 3(a). This reflects the symmetry of the underlying Hamiltonian and robustness of our simulation to the imperfections in the annealing device. While not explicitly compared here, we generally find that our magnetization results from QA are similar to magnetic susceptibility measurements observed in Shastry-Sutherland candidate materials such as the rare-earth tetraborides [9,23,24]. Moreover, real material synthesis is susceptible to defect formation, which indicates a potentially beneficial connection between the defects present in both the QA hardware and the defects in single crystals of candidate ShastrySutherland materials.

Quantum annealing also allows us to identify the critical regimes for the phase transitions. These are indicated in Fig. 3(b) by the white stripes between the ordered phases, corresponding to where the derivative of the magnetization is largest. The critical regimes are locations where the long-range spin order breaks down, leading to spin textures and an increased sensitivity to defects [25]. A real-space examination of this result from the QA processor is presented in Figs. 3(c)-3(f), showing the microscopic ordering expected in the different phases as we tune the longitudinal field. The location of these structures are indicated in Fig. 3(b). We show that in Fig. 3(c) we obtain the typical Néel AFM ordering with zero average magnetization. In Fig. 3(d) we display the spin texture at the critical point from the Néel phase, showing clearly the increasing sensitivity to defects. These defects act as nucleation points for the horizontal FM chains that are precursors of the $\frac{1}{3}$ plateau phase. 


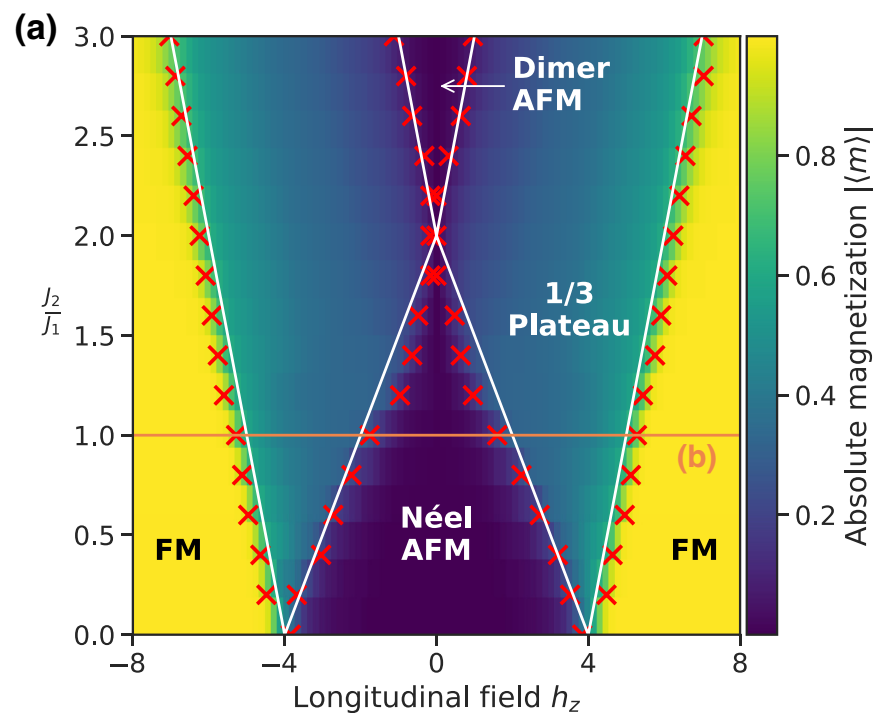

(c)

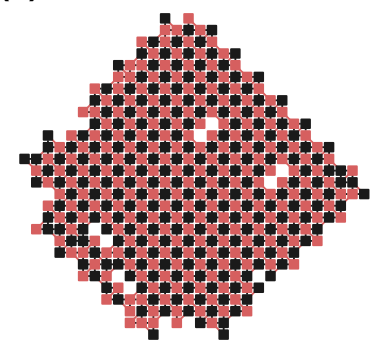

(d)

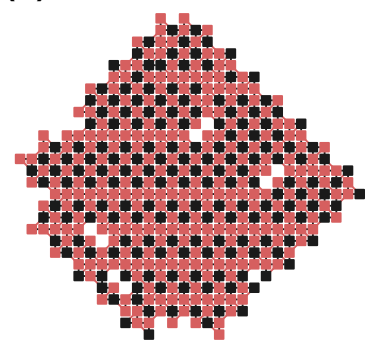

(b)

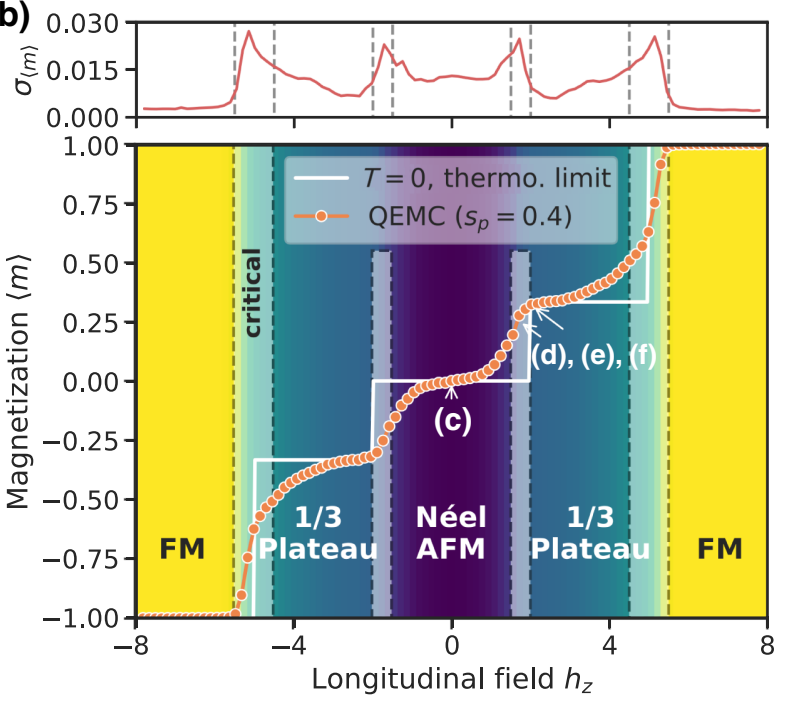

(e)

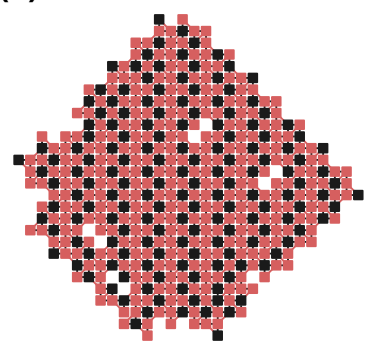

(f)

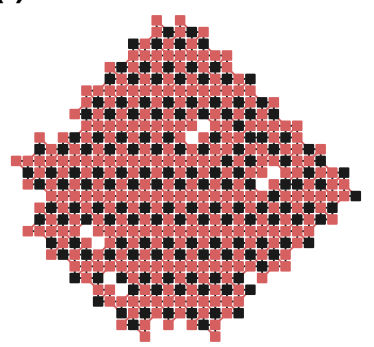

FIG. 3. Phase diagram and spin structures. (a) Phase diagram obtained by QEMC chains with $t_{r}=1 \mu \mathrm{s}, t_{p}=1998 \mu \mathrm{s}$, and $s=0.4$. The simulated phase boundaries, shown with red crosses, are determined by calculating the point of steepest slope of magnetization. This helps to distinguish the $\frac{1}{3}$ plateau, the dimer, FM, and the Néel states. The white lines outline the exact phase diagram as determined analytically by Dublenych [6]. A slice of the phase diagram at $J_{2} / J_{1}=1$ is shown in (b). (b) Slice of phase diagram at $\left(J_{1}=1, J_{2}=1\right)$ obtained by QEMC chains with $t_{r}=1 \mu \mathrm{s}, t_{p}=1998 \mu \mathrm{s}$, and $s=0.4$. The observed phase transition is broadened compared to exact results due to a number of compounding effects, the most notable being bond disorder of $J_{1}, J_{2}$, residual effects of point defects and finite lattice size, non-negligible persistent transverse field, and temperature effects. Each data point is the average of 100 QEMC chains, each of 100 iterations in which we sample the last 50, providing 5000 spin configurations per data point. We observe that the standard deviation of the magnetization, $\sigma_{\langle m\rangle}$, lies within the range $0.002 \leq \sigma_{\langle m\rangle}<0.03$ and has characteristic peaks within the critical regimes. We find that $\sigma_{\langle m\rangle}$ is sufficiently small that the round markers obscure the plotted error bars completely and so it is plotted above the magnetization for clarity. Four points are labeled (c)-(f) that correspond to the structures shown in panels (c)-(f). The critical regimes are highlighted in white. (c),(d) The real-space spin motifs determined by QEMC chains in the Néel AFM and critical AFM to plateau phase calculated at the points labeled in (b). In (d) we observe a coexistence between the Néel and plateau orderings separated by domain walls. (e),(f) Two degenerate solutions within the plateau phase. The black and red squares represent spin up and down, respectively.

In Figs. 3(e) and 3(f) we display two of these six observed degenerate spin structures found within the plateau phase, each with $\langle m\rangle=\frac{1}{3}$. In our various QA simulations we are able to recover all six degenerate solutions for the $\frac{1}{3}$ plateau. Additionally, the spin textures around the defects (white dots) are obvious in Figs. 3(c)-3(f). Deeper in the $\frac{1}{3}$ phase the presence of the defects does not destroy the local magnetic Hamiltonian, which speaks to the effectiveness of the mean-field boundary conditions. Our simulations predict that one should expect a different degeneracy of the $\frac{1}{3}$ plateau solution around the defects in small domains as shown in
Figs. 3(d) and 3(f) in the complete absence of boundary conditions.

The microscopic real-space structures obtained directly from the QA method can be verified using magnetic microscopy (such as Lorentz-force microscopy); however, such techniques are challenging for 2D materials in a magnetic field. A preferred experimental technique for studying magnetic ordering is in the Fourier space via measurement of the static structure factor obtained from diffuse neutron scattering. To elucidate the connection between our annealing results with this experimental technique, we compute the static structure factor 

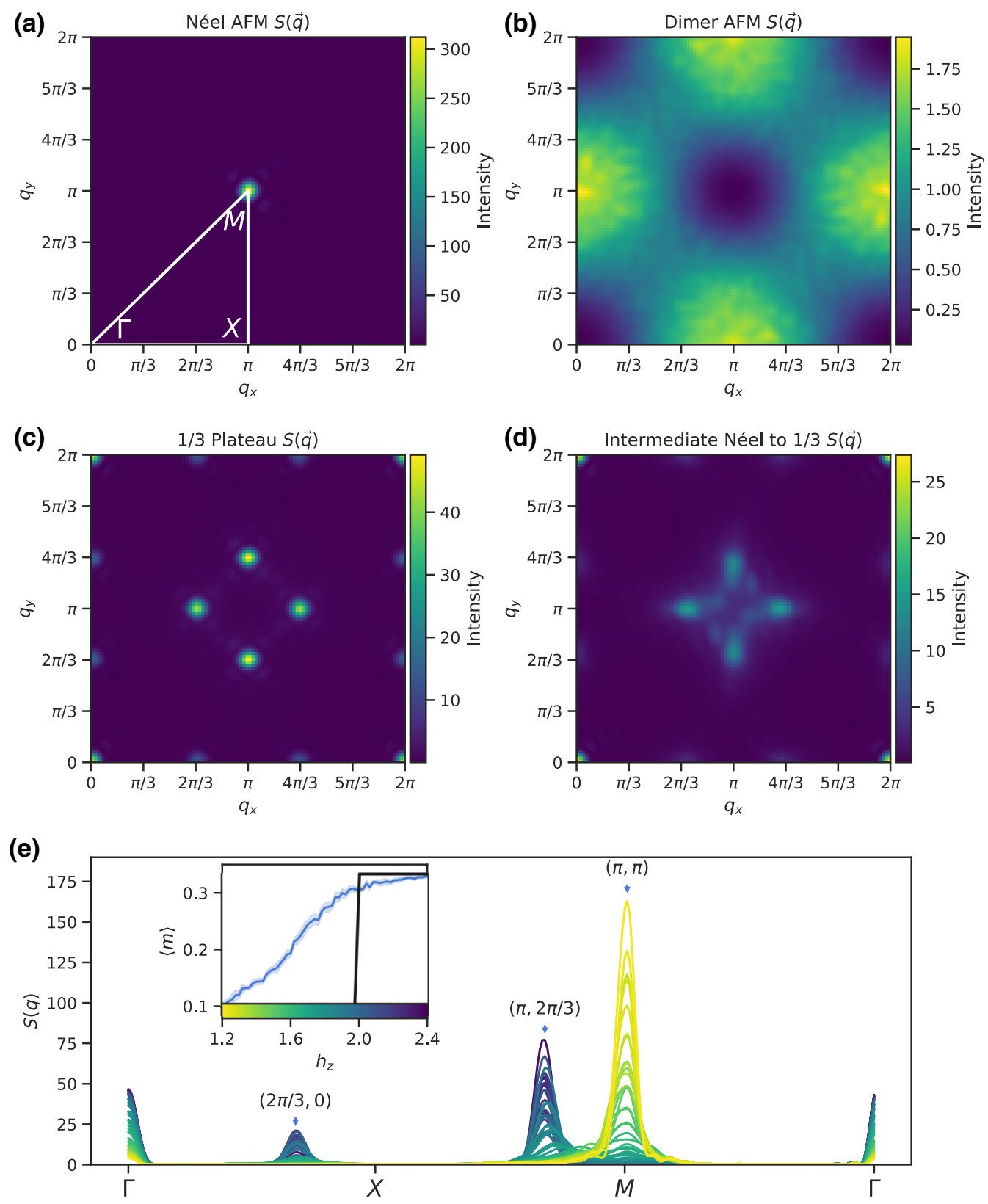

FIG. 4. Static structure factors in equilibrium and at criticality. (a) Structure factor of the Néel AFM phase at $J_{1}=J_{2}=1, h_{z}=0$. The high symmetry points of this model are labeled in white along with a cut path used in (e); these points are $\left(q_{x}=0, q_{y}=0\right)$, $\left(q_{x}=\pi, q_{y}=0\right)$, and $\left(q_{x}=\pi, q_{y}=\pi\right)$. (b) Structure factor of the dimer AFM phase at $J_{1}=1, J_{2}=3, h_{z}=0$. (c) Structure factor of the $\frac{1}{3}$ plateau phase at $J_{1}=J_{2}=1, h_{z}=2.1$. (d) Structure factor in the transition between Néel AFM and $\frac{1}{3}$ plateau phases at $J_{1}=J_{2}=1, h_{z}=1.8$. (e) A cut along the symmetry points shown in (a) of the structure factor through the Néel to plateau transition. The inset figure shows the color scale corresponding to the longitudinal field across the phase transition at $J_{2} / J_{1}=1$.

$S(\vec{q})$, defined in terms of the two-point correlation function as

$$
S(\vec{q})=\sum_{i, j}\left\langle\sigma_{(i)}^{z} \sigma_{(j)}^{z}\right) e^{i \vec{q} \cdot\left(\vec{R}_{i}-\vec{R}_{j}\right)}
$$

where $\vec{R}_{i j}$ is the relative position of two spins and $\vec{q}$ is the wave vector in reciprocal space. The static structure factor provides a Fourier decomposition of the spatial correlations encoded by the spin system and quantifies the ordering of different magnetic phases. The calculated correlations for the Néel AFM, dimer AFM, and $\frac{1}{3}$ plateau phase are shown in Figs. 4(a)-4(c) and agree with theoretical expectations [6]. Because the static structure factor is directly measured by the neutron diffuse scattering spectrum, we anticipate that similar QA results 
for appropriately modified Hamiltonians will enable comparisons with real Shastry-Sutherland magnets in the future.

Unlike the standard forward-annealing use of QA, QEMC chains allow us to probe ordering and statistical convergence near critical regimes [14], including, for example, the first-order transition from the Néel AFM to the $\frac{1}{3}$ plateau. However, while theory predicts a sharp discontinuity in the magnetization, our annealing simulations produce a more gradual change, as shown in Fig. 4(e). We surmise that the origin of this smoothing includes bond disorder in $J_{1}, J_{2}$ that is intrinsic to the annealer and uncorrected by our boundary conditions, residual effects from point defects, and finite lattice size, as well as non-negligible transverse-field effects persisting near the phase transition. It has also been shown in previous work that temperature can contribute to smoothing the phase transitions [7]. However, the relative strength of thermal to coupling energies used in this device $\left[k_{B} T(14 \mathrm{mK}) /\left\{J_{1} B(s=1)\right\} \approx 0.02\right]$ was found to be sufficiently small to ignore the finite temperature broadening observed in Ref. [7].

We compute the structure factors near the critical regime from the Néel to plateau phases, where intermediate spin structures are observed that do not exist in either phase. Sweeping through the critical regime yielded a smoothed interpolation between the Néel and plateau structures; examining the structure factor at an intermediate point in this transition enables us to predict the spin structures and their spatial correlations near criticality. Shown in Fig. 4(d) is the structure factor corresponding to the spin texture in Fig. 3(d) at the critical point between the Néel and plateau phases. This reveals an asymmetric broadening of the peaks in the static structure factor, reminiscent of fluctuations across length scales - phenomena observed experimentally in continuous phase transitions (see, e.g., Ref. [26]) - revealing a pathway to characterize complex shortrange correlated physics near a phase boundary, physics that is extremely relevant to novel spin ice materials [3]. Our results show that the current generation of QA has the maturity to explore critical phenomena in frustrated magnets and predict experimentally accessed results relevant to a variety of spintronic materials, opening a new paradigm for QA.

\section{CONCLUSIONS}

To conclude, we have used the Shastry-Sutherland model to successfully demonstrate how QA may be used to investigate the short-ranged, highly degenerate states and phase diagrams in a general class of frustrated magnetic systems. This demonstration required addressing hardware limitations such as defects and finite-size effects, in addition to optimization of the annealing protocol. Specifically, we apply novel boundary conditions and a tunable annealing protocol, known as QEMC chaining, to simulate the low-energy manifold of a complex Ising Hamiltonian over a range of model parameters. The fidelity of our simulations further demonstrates that qubit and bond imperfections inherent in near-term annealers, as well as limited system control, can be effectively mitigated by taking advantage of other programmable features of QA devices. Taken together with the computation of the structure factor, QA provides a new utility for predicting the emergence of spin behavior in statistical systems across length scales.

This work indicates that experimental data analyses based on quantum annealing simulations of complex Hamiltonians is no longer a conceptual challenge, but rather a technical one and we anticipate that these results coupled with next-generation annealers [27] will play an important role in the future of condensed matter physics. This work opens a route for a practical application of qubit-based quantum annealers in material physics applications and new possibilities for understanding real materials using quantum hardware in ways that have historically been difficult or impossible to achieve.

The Department of Energy will provide public access to these results of federally sponsored research in accordance with the DOE Public Access Plan [28].

\section{ACKNOWLEDGMENTS}

We thank M.A. McGuire and J.-Q. Yan of Oak Ridge National Laboratory for insightful comments on fractional magnetic materials. We also thank C.D. Batista and D.A. Tennant of Oak Ridge National Laboratory for feedback on this work's impact and scope. This work is supported by the Department of Energy, Office of Science, Early Career Research Program. This research used quantum computing resources of the Oak Ridge Leadership Computing Facility, which is a DOE Office of Science User Facility supported under Contract No. DE-AC05-00OR22725. Work performed by A.B. while at SNS is supported by the DOE Office of Science User Facilities Division. This manuscript has been authored by UT-Battelle, LLC under Contract No. DE-AC05-00OR22725 with the U.S. Department of Energy. The U.S. Government retains, and the publisher, by accepting the article for publication, acknowledges that the U.S. Government retains a nonexclusive, paid-up, irrevocable, worldwide license to publish or reproduce the published form of this manuscript, or allows others to do so, for U.S. Government purposes.

\section{APPENDIX A: EMBEDDING}

The D-Wave 2000Q quantum annealer is constructed of superconducting flux qubits coupled together in the Chimera topology [20]. The quantum annealer has two 


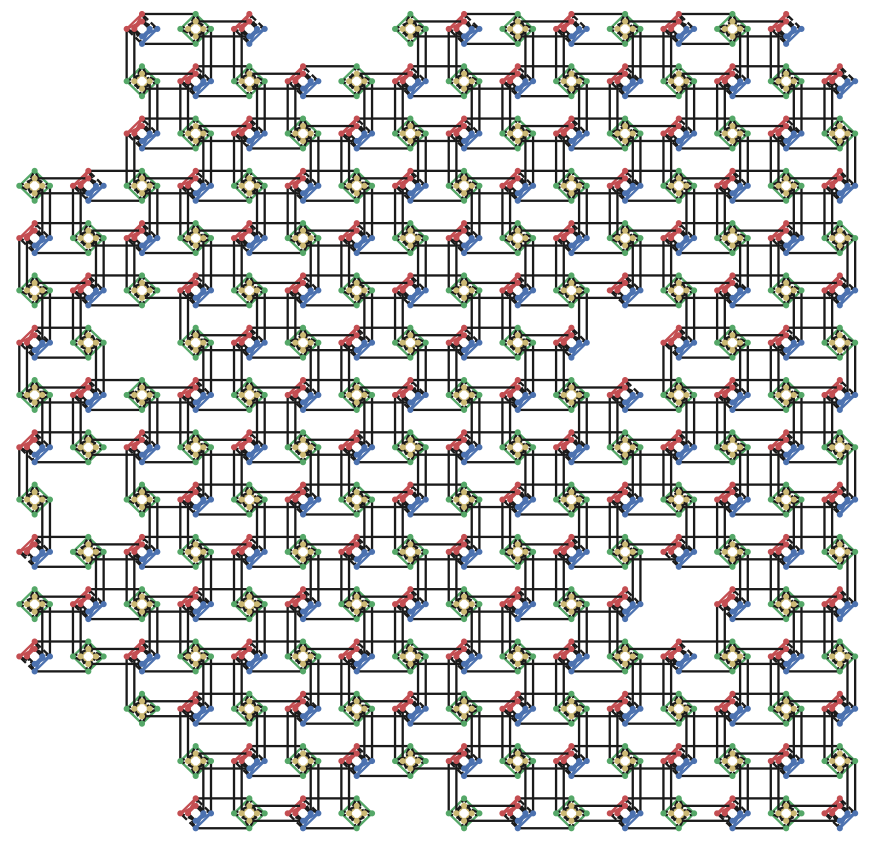

FIG. 5. Half-cell embedding into the D-Wave quantum annealer with unit cell as depicted in Fig. 1(b). This is rotated $45^{\circ}$ to obtain the lattice shown in Fig. 1(c).

types of couplers: internal unit-cell couplers, which control interactions within a unit cell, and external couplers, which mediate interactions between unit cells. Here we present an embedding, shown in Figs. 1 and 5, which places one logical dimer onto each Chimera unit cell and realizes square bonds between dimers through intercell Chimera couplers. This embedding leverages the natural structure of the quantum annealer architecture to reinforce the symmetries of the logical model.

Sometimes the ferromagnetic chains that represent a single logical spin become misaligned - this is called a chain break - which often leads to incorrect logical solutions. The observed probability for a chain break is estimated (from the phase diagram data shown in Fig. 3) to be less than $0.02 \%$ and, therefore, we do not employ methods for decoding embedded chains to logical values, but rather use the first physical spin (determined by the smallest linear qubit index) of each chain as representative of the logical spin. While this does introduce some additional error, the contribution is very small relative to other sources of noise. The low probability of chain breaking is due primarily to the cyclic nature of the chains and the symmetry preservation of the embedding.

\section{APPENDIX B: CHI-COMPENSATION}

The radio-frequency superconducting quantum interference device flux qubits in the D-Wave 2000Q system do not perfectly implement the TFIM Hamiltonian.
Specifically, each qubit mediates an effective coupling between neighboring qubits regardless of the existence of a physical coupler. Additionally, there is leakage of an applied $h$ bias from a qubit to its neighbors. The strength of these additional effects are dependent on the normalized background susceptibility $\chi_{b}=M_{\mathrm{AFM}} \chi_{q}$, where $M_{\mathrm{AFM}}$ is the maximum available AFM mutual inductance and $\chi_{q}$ is the physical qubit susceptibility. These effects lead to a first-order modification to the embedded $h$ and $J$ :

$$
\begin{aligned}
J_{i, k}\left(\chi_{b}\right) & =J_{i, k}+\sum_{j} \chi_{b} J_{i, j} J_{j, k}, \\
h_{i}\left(\chi_{b}\right) & =h_{i}+\sum_{j} \chi_{b} J_{i, j} h_{j} .
\end{aligned}
$$

For the low-noise D-Wave 2000Q system used in this work, $\chi_{b}$ varies as a function of the anneal parameter $s$, for the regime of $0.4<s<0.6$ probed $\chi_{b}=-0.03 \pm 0.01$. Along with the $J_{1}, J_{2}, h$ parameters, $J_{3}$ is the strength of the FM chains that describe a single logical spin, and is fixed in all experiments to $J_{3}=-1$. For the half-cell embedding presented here, it is sufficient to derive expressions of the impact of these interactions on the logical problem:

$$
\begin{aligned}
J_{2}\left(\chi_{b}\right) & =J_{2}\left(1+4 \chi_{b} J_{3}\right), \\
J_{1}\left(\chi_{b}\right) & =J_{1}\left(1+4 \chi_{b} J_{3}+2 \chi_{b} J_{2}\right), \\
h\left(\chi_{b}\right) & =h\left(1+\chi_{b} J_{1}+2 \chi_{b} J_{2}+2 \chi_{b} J_{3}\right) .
\end{aligned}
$$

We use these expressions to determine the correct input such that the embedded system corresponds to the correct logical problem instance.

\section{APPENDIX C: FLUX-BIAS OFFSET CALIBRATION}

The D-Wave 2000Q system is calibrated as a quantum annealer to perform well under a variety of input types, and the unique problem here permits the recalibration of the device for improved performance. This calibration should be done to reinforce the expected symmetries in the system, and in the absence of an applied longitudinal field the system is invariant under a spin-flip operation. Using this symmetry, we determine flux-bias offsets for each qubit with a gradient descent method such that the average qubit magnetization is zero. The measurement of this magnetization is performed via forward anneals in this work but is generally a function of anneal schedule.

\section{APPENDIX D: MEAN-FIELD BOUNDARY CONDITIONS}

Because of the nature of the half-cell embedding, any missing qubit or coupler on the D-Wave 2000Q chip leads to a missing qubit or coupler in the logical 
Shastry-Sutherland graph, respectively. Because of the finite size of the chip and the presence of these defects, appropriate boundary conditions must be chosen in order to recover the expected solutions in the thermodynamic limit.

Here we implement a form of mean-field boundary conditions $[18,19]$ that are found via an optimization over the longitudinal magnetic fields of qubits on external and internal boundaries. Specifically, we seek to determine the appropriate magnetic fields such that the magnetization of boundary qubits is equivalent to the magnetization of bulk qubits and is defined as

$$
\begin{gathered}
\min _{\vec{h}_{\text {bound }}}\langle m\rangle_{\text {bulk }}-\langle m\rangle_{\text {bound }} \\
\text { such that } \operatorname{sign}\left(\vec{h}_{\text {bound }}\right)=\operatorname{sign}\left(h_{\text {bulk }}\right),
\end{gathered}
$$

where $\vec{h}_{\text {bound }}$ is the vector of longitudinal magnetic fields on the boundary and $\langle m\rangle_{\text {bulk }},\langle m\rangle_{\text {bound }}$ are the magnetizations of the bulk and boundary qubits, respectively. This ensures homogeneous magnetization between interior and boundary qubits, which reinforces the translational invariance of the order parameter. The sign constraint is used to restrict the boundary fields to physically realistic local environments.

The optimization is done via a simple gradient descent method that updates each boundary longitudinal field according to

$$
h_{i}^{t+1}=h_{i}^{t}+\delta h\left(\left\langle\sigma_{(i)}^{z}\right\rangle-\langle m\rangle_{\text {bulk }}\right),
$$

where $\left\langle\sigma_{(i)}^{z}\right\rangle$ is the average magnetization of qubit $i$ over all samples and $0 \leq \delta h \leq 0.05$ is the step size. We typically see convergence of this parameterization up to statistical noise in at most 400 iterations. Some points used additional iterations and served as a check that 400 is a sufficient maximum number of iterations. We observed no significant correlation between the iteration count and problem parameters.

\section{APPENDIX E: QUANTUM EVOLUTION MONTE CARLO CHAINS}

In quantum annealing the goal is to evolve a system adiabatically from an eigenstate of the transverse field at $s=0$ to an eigenstate of the Ising model at $s=1$. However, for systems composed of chains of ferromagnetically coupled qubits, there may be a value of $s$ at which the spins of the chain fail to evolve meaningfully and freeze [22]. This freeze out point is a function of the average length of the chains and, for chains of four qubits, the freeze out point for this annealer is approximately $s=0.4$, where the transverse field is non-negligible. If the system freezes near this position in the anneal then the collected measurement statistics will not resemble a classical Ising model but the
TFIM. Shown in Fig. 6(a) is the relative energy scale of the individual terms in the Hamiltonian equation (2) as a function of $s$ compared to the characteristic thermal energy of the system.

Here we adapt a technique used by King et al. [17] and chain together a sequence of QEMC steps, individually referred to as reverse anneals. In a reverse anneal the system is initiated in a classical state at $s=1$, follows an anneal schedule, and then is read out to obtain a new classical state. The anneal schedule used here [see Fig. 6(b)] rapidly reverse anneals to a point in the anneal schedule with non-negligible transverse field, pauses to allow the system to thermalize and populate low-energy intermediate states, and forward anneals to finish the protocol. It is not immediately obvious that this anneal schedule will allow us to probe classical states; however, since each lattice site is represented by a cyclic chain of four physical qubits, the effective tunneling in the logical model is much smaller than the tunneling of individual qubits, suggesting that at $s_{p}=0.4$ the system is still sufficiently classical with a weak transverse field. In the Shastry-Sutherland Ising model, where even in the dimer phase, ground states are separated by two logical spin flips. The relevant physical tunneling is therefore not a four-qubit term but an eightqubit term, which is very small even when the transverse field is moderate, as in our situation.

When repeated many times, this protocol resembles a Markov chain Monte Carlo protocol and enables the determination of ground states through an iterative refinement of solutions shown in Fig. 6(c). This repeated process resembles the Markov chain Monte Carlo method, but fails to be perfectly Markovian due to correlations between iterations. Just as in a conventional Markov chain simulation, we discard the first half of the iterations as burn-in steps while the system equilibrates and then sample from the last half of the chain. The chain length size depends greatly on the pause point $s_{p}$, as can be seen in Fig. 2(b).

Additionally, during long pause times we do not expect to completely prepare a quantum thermal state of the instantaneous device Hamiltonian $H\left(s_{p}\right)$, although we do expect to approach it. In fact, we know that we do not prepare quantum thermal states because if we did we would observe that the QEMC chain converges instantly because in a quantum thermal state (i.e., at equilibrium) all information about the initial state should be lost, which is not the case in our results, as seen in Fig. 2(b). So we prefer a faster forward anneal $t_{r}$ to permit a longer pause, which is shown in Fig. 2(c) to result in lower energy states. Because of the experimental constraints, we are unable to probe the trade-off between pause and forward-anneal times at longer anneal times.

The strength of the transverse field is determined by the pause position $s_{p}$, and a value of $s_{p} \approx 0.4$ returns an ensemble of states with minimal experimental variance and the lowest observed energies over all experiments. For 


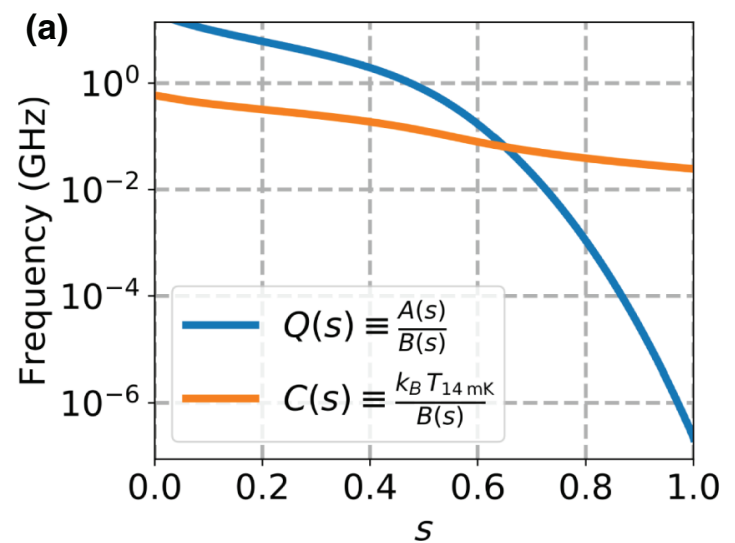

(b)

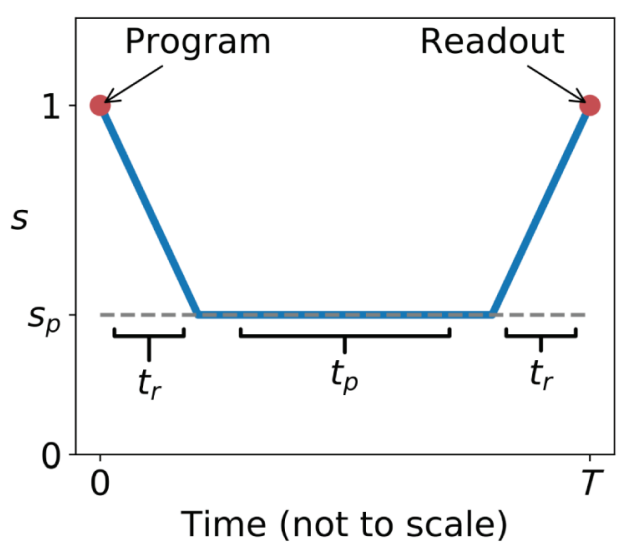

(c)

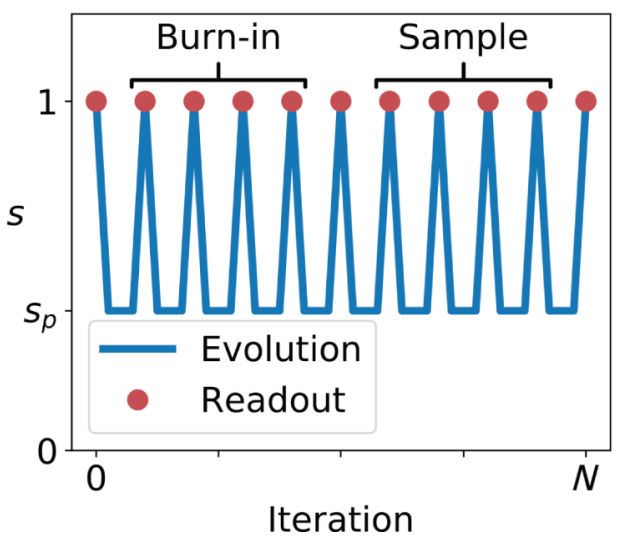

FIG. 6. (a) The annealing parameter $s$ controls amplitudes $A(s)$ and $B(s)$ in the device Hamiltonian, Eq. (2). Here $Q(s)$ quantifies the relative strengths of these terms as a function of $s$. Also shown is the dependence on $s$ of the ratio to amplitude $B(s)$ and the characteristic thermal energy of the system at the operating temperature of $14 \mathrm{mK}$. (b) A single QEMC anneal schedule that shows the initialization of the annealer with an eigenstate of the classical Hamiltonian $[A(s) \ll B(s)]$ at $t=0$, reverse annealing for time $t_{r}$, pausing for time $t_{p}$ at $s_{p}$ [where $Q(s) \geq 1$ ], and forward annealing for time $t_{r}$. (c) An iterative protocol where multiple QEMC steps are chained together by reprogramming the next QEMC step with the output from the previous QEMC step. The first half of the samples are discarded as burn-in steps while the chain equilibrates and the last half of the samples are collected and used for analysis. $s>0.4$, we find that the solution quality declines rapidly because the transverse field is insufficient to drive tunneling through energy barriers, while for stronger transverse fields $(s<0.4)$, we observe that the sampling statistics are distorted by the transverse field. As shown in Fig. 2(b), higher transverse-field strengths increased the rate of convergence, but converged to an ensemble with higher average energy relative to those observed at $s_{p}=0.4$. As shown in Fig. 2(c), the prepared ensembles are found to be sensitive to the pause duration $t_{p}$ in the range of $1-1998 \mu \mathrm{s}$ for $s_{p}=0.4$.

All experiments performed in this work used the same reverse anneal time $t_{r}=1 \mu \mathrm{s}$ and used a chain length of 100 , so that 50 steps are used for chain equilibration and 50 states are used for statistical sampling. For the system under study, the timescale of dynamics is assumed to be much larger than the timescale of the anneal and readout; therefore, a rapid forward anneal permits more time to pause and thermalize while not populating high-energy states during the forward anneal. Finally, the initial state to begin each QEMC chain in this work is chosen to be the FM all spin-up state. We find little dependence of the final state of the chain on the initial state if $s_{p}$ is small enough such that the transverse field is significantly strong $\left(s_{p} \leq 0.6\right)$, as can be seen in Fig. 2(b).

During the pause at $s_{p}$ the persistent current flowing in the superconducting device generates a magnetic field that acts to polarize the ensemble of spins, an effect called spinbath polarization (SBP). This effect is typically stronger for long pause times; however, in this work we have used a DWave 2000Q lower-noise processor, which has roughly 1.5 times to 2 times less midband noise than the processor used in the SBP measurements in Ref. [29] (these fabrication processes are FAB2-INT and FAB2 in the D-Wave white paper [30]). In our experiments we insert a depolarization time (also known as "readout thermalization") of $100 \mu \mathrm{s}$, which significantly lowers the SBP effect between anneals. Additionally, each data point in this work is the average over multiple QEMC chains, and the timescales between QEMC chains is long enough to nullify any intercall spinbath polarization effect.

[1] C. Broholm, R. J. Cava, S. A. Kivelson, D. G. Nocera, M. R. Norman, and T. Senthil, Quantum spin liquids, Science 367, 6475 (2020).

[2] S. T. Bramwell, Spin ice state in frustrated magnetic pyrochlore materials, Science 294, 1495 (2001).

[3] K. Zhao, H. Deng, H. Chen, K. A Ross, V. Petrríček, G. Günther, M. Russina, V. Hutanu, and P. Gegenwart, Realization of the kagome spin ice state in a frustrated intermetallic compound, Science 367, 1218 (2020).

[4] Q. Wang, Y. Shen, B. Pan, Y. Hao, M. Ma, F. Zhou, P. Steffens, K. Schmalzl, T. R. Forrest, M. Abdel-Hafiez, et al., Strong interplay between stripe spin fluctuations, 
nematicity and superconductivity in fese, Nat. Mater. 15, 159 (2016).

[5] B. S. Shastry and B. Sutherland, Exact ground state of a quantum mechanical antiferromagnet, Phys. B+ C 108, 1069 (1981).

[6] Yu. I. Dublenych, Ground States of the Ising Model on the Shastry-Sutherland Lattice and the Origin of the Fractional Magnetization Plateaus in Rare-Earth-Metal Tetraborides, Phys. Rev. Lett. 109, 167202 (2012).

[7] M.-C. Chang and M.-F. Yang, Magnetization plateau of the classical Ising model on the shastry-sutherland lattice: A tensor renormalization-group approach, Phys. Rev. B 79, 104411 (2009).

[8] J. Trinh, S. Mitra, C. Panagopoulos, T. Kong, P. C. Canfield, and A. P. Ramirez, Degeneracy of the 1/8 Plateau and Antiferromagnetic Phases in the Shastry-Sutherland Magnet tmb 4 , Phys. Rev. Lett. 121, 167203 (2018).

[9] K. Siemensmeyer, E. Wulf, H.-J. Mikeska, K. Flachbart, S. Gabáni, S. Mat'aš, P. Priputen, A. Efdokimova, and N. Shitsevalova, Fractional Magnetization Plateaus and Magnetic Order in the Shastry-Sutherland Magnet tmb $b_{4}$, Phys. Rev. Lett. 101, 177201 (2008).

[10] W. C. Huang, L. Huo, G. Tian, H. R. Qian, X. S. Gao, M. H. Qin, and J. M. Liu, Multi-step magnetization of the Ising model on a shastry-sutherland lattice: A monte carlo simulation, J. Phys.: Condens. Matter 24, 386003 (2012).

[11] S. Kirkpatrick, C. D. Gelatt, and M. P. Vecchi, Optimization by simulated annealing, Science 220, 671 (1983).

[12] J. Brooke, D. Bitko, and G. Aeppli, Quantum annealing of a disordered magnet, Science 284, 779 (1999).

[13] M. W. Johnson, M. H. S. Amin, S. Gildert, T. Lanting, F. Hamze, N. Dickson, R. Harris, A. J. Berkley, J. Johansson, P. Bunyk, et al., Quantum annealing with manufactured spins, Nature 473, 194 (2011).

[14] A. D. King, J. Raymond, T. Lanting, S. V. Isakov, M. Mohseni, G. Poulin-Lamarre, S. Ejtemaee, W. Bernoudy, I. Ozfidan, A. Y. Smirnov, et al., Scaling advantage in quantum simulation of geometrically frustrated magnets, arXiv:1911.03446 (2019).

[15] T. Boothby, A. D. King, and A. Roy, Fast clique minor generation in chimera qubit connectivity graphs, Quantum Inf. Process. 15, 495 (2016).

[16] R. Harris, Y. Sato, A. J. Berkley, M. Reis, F. Altomare, M. H. Amin, K. Boothby, P. Bunyk, C. Deng, C. Enderud, et al., Phase transitions in a programmable quantum spin glass simulator, Science 361, 162 (2018).

[17] A. D. King, J. Carrasquilla, J. Raymond, I. Ozfidan, E. Andriyash, A. Berkley, M. Reis, T. Lanting, R. Harris, F. Altomare, et al., Observation of topological phenomena in a programmable lattice of 1800 qubits, Nature 560, 456 (2018).

[18] H. Müller-Krumbhaar and K. Binder, A "self consistent" monte carlo method for the heisenberg ferromagnet, $\mathrm{Z}$. Phys. A Hadrons Nucl. 254, 269 (1972).

[19] D. P. Landau and K. Binder, A Guide to Monte Carlo Simulations in Statistical Physics (Cambridge University Press, Cambridge, 2014).

[20] P. I. Bunyk, E. M. Hoskinson, M. W. Johnson, E. Tolkacheva, F. Altomare, A. J. Berkley, R. Harris, J. P. Hilton, T. Lanting, A. J. Przybysz, et al., Architectural considerations in the design of a superconducting quantum annealing processor, IEEE Trans. Appl. Supercond. 24, 1 (2014).

[21] V. Choi, Minor-embedding in adiabatic quantum computation: I. The parameter setting problem, Quantum Inf. Process. 7, 193 (2008).

[22] M. H. Amin, Searching for quantum speedup in quasistatic quantum annealers, Phys. Rev. A 92, 052323 (2015).

[23] D. Brunt, G. Balakrishnan, A. R. Wildes, B. Ouladdiaf, N. Qureshi, and O. A. Petrenko, Field-induced magnetic states in holmium tetraboride, Phys. Rev. B 95, 024410 (2017).

[24] S. Mat'aš, K. Siemensmeyer, E. Wheeler, E. Wulf, R. Beyer, Th. Hermannsdörfer, O. Ignatchik, M. Uhlarz, K. Flachbart, S. Gabáni, P. Priputen, A. Efdokimova, and N. Shitsevalova, Magnetism of rare earth tetraborides, J. Phys.: Conf. Ser. 200, 032041 (2010).

[25] James Sethna, Statistical Mechanics: Entropy, Order Parameters, and Complexity (Oxford University Press, Oxford, 2006), Vol. 14.

[26] Y. Feng, J. Wang, R. Jaramillo, J. van Wezel, S. Haravifard, G. Srajer, Y. Liu, Z.-A. Xu, P. B. Littlewood, and T. F. Rosenbaum, Order parameter fluctuations at a buried quantum critical point, Proc. Natl. Acad. Sci. 109, 7224 (2012).

[27] Kelly Boothby, Paul Bunyk, Jack Raymond, and Aidan Roy, Next-Generation Topology of D-Wave Quantum Processors. arXiv:2003.00133 [quant-ph] (February 2020).

[28] http://energy.gov/downloads/doe-public-access-plan.

[29] T. Lanting, M. H. Amin, C. Baron, M. Babcock, J. Boschee, S. Boixo, V. N. Smelyanskiy, M. Foygel, and A. G. Petukhov, Probing environmental spin polarization with superconducting flux qubits, arXiv:2003.14244 (2020).

[30] Probing mid-band and broad-band noise in lower-noise d-wave 2000q fabrication stacks. https://www.dwavesys.

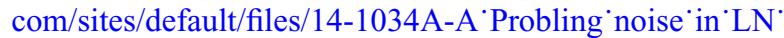
2000Q fabrication'stacks.pdf. Accessed: 2020-10-5. 